Research Article

\title{
Condition-Based Maintenance Optimization for Motorized Spindles Integrating Proportional Hazard Model with SPC Charts
}

\author{
Xuejiao Du $\mathbb{D}^{1},{ }^{1}$ Jingbo Gai $\mathbb{D}^{1},{ }^{1}$ and Cen Chen $\left.{ }^{2}\right)^{2}$ \\ ${ }^{1}$ College of Aerospace and Civil Engineering, Harbin Engineering University, Harbin 150001, China \\ ${ }^{2}$ Department of Electrical Engineering, Harbin Institute of Technology, Harbin 150001, China
}

Correspondence should be addressed to Xuejiao Du; duxuejiao@hrbeu.edu.cn

Received 22 May 2020; Accepted 29 June 2020; Published 21 July 2020

Academic Editor: Francesco Pellicano

Copyright (c) 2020 Xuejiao Du et al. This is an open access article distributed under the Creative Commons Attribution License, which permits unrestricted use, distribution, and reproduction in any medium, provided the original work is properly cited.

Reliability of motorized spindles has a great effect on the performance and productivity of computer numerical control (CNC) machine tools for intelligent manufacturing. Condition-based maintenance (CBM) is an efficient method to prevent serious failures, to improve system reliability, and to reduce management costs for motorized spindles. However, owing to various degradation features acquired during condition monitoring, the challenge is to propose an appropriate feature to evaluate the reliability level of motorized spindles and to set up optimal CBM policies. Based on the motivation, a three-stage approach is proposed in this paper. In the first stage, proportional hazard model (PHM) is developed to describe the reliability considering failure events together with multiple degradation features. Next, statistical process control (SPC) charts are constructed for condition monitoring and anomaly detection in order to achieve early detection of potential failures. At last, a CBM schedule is modeled in consideration of maintenance cost minimization; the maintenance plan is optimized by determining the optimal control limits of SPC charts.

\section{Introduction}

As an emerging technology in the field of intelligent manufacturing, the motorized spindle of the computer numerical control (CNC) machine tool is regarded as a core component of all manufacturing facilities; its performance could determine the machining accuracy and production efficiency of CNC machine tools, even the whole manufacturing supply chain, to a large extent [1]. Thus, its reliability improvement and health management cause widespread concern. For the purpose of improving the availability and reducing production expenses of the motorized spindle, condition-based maintenance (CBM) has drawn much attention [2]. It focuses on utilizing the condition monitoring information collected in real time to assess health status and to develop optimal maintenance strategies [3].

As a condition monitoring method, control charts of the statistical process control (SPC) theory can be incorporated with maintenance analysis to realize CBM optimization.
Shrivastava et al. [4] integrated the cumulative sum (CUSUM) control chart with preventive maintenance (PM) planning for joint optimization of maintenance activities and chart parameters. Integrated models combining the $\bar{X}$ control chart with PM actions were proposed in $[5,6]$ in order to protect the equipment from quality decline. Lampreia et al. [7] modified CUSUM and exponentially weighted moving average (EWMA) control charts for online vibration monitoring, thus reducing false alarms and planning early interventions. The similar modelling idea was also adopted for the economic design of maintenance plans with the Shewhart control chart, chi-square control chart, etc., as discussed in [8-12]. Mehrafrooz and Noorossana [13] developed a model under the assumption of perfect maintenance; the model could express six alternative scenarios of the monitored process to achieve the most economic maintenance schedule. The integrated model was further developed by Yin et al. [14] considering ten scenarios of the delayed monitoring process. From the perspective of reliability improvement, some SPC charts were also established 
to monitor the feature of time-between-events (TBE) assuming that TBE followed the gamma distribution [15], the normal distribution [16], the exponential distribution [17], the Weibull distribution [18], etc.

However, these aforementioned control charts are all univariate, which are commonly used to monitor individual observations of a certain type of characteristic variable. For the case of the motorized spindle, condition monitoring usually contains multiple sensor signals, such as vibration, bearing preload, acoustic emission, sound, and temperature [19], and the signals are often autocorrelated [20]. Therefore, the conventional SPC charts will not be effective to extract useful and reliable information from the monitoring process, leading to larger errors in CBM optimization.

To overcome this problem, the paper develops a threestage approach for the purpose of extracting the fusion feature, monitoring the health degradation, and optimizing the CBM strategy for motorized spindles. The main contributions of the proposed approach are described as follows:

(1) Proportional hazard model (PHM) is introduced to extract the health indicator. The benefits can be demonstrated in two aspects: on the one hand, PHM can integrate multivariate information from multiple monitoring signals together with failure data, which results in the more accurate degradation detection for CBM optimization. On the other hand, the hazard rate from PHM is selected as the health indicator of SPC charts. It can reduce random interference for SPC charts when monitoring the autocorrelation process; the detection error, such as the false alarm or undetected failure, can be effectively avoided.

(2) CBM optimization is combined with SPC chart design based upon economic considerations, the health condition of motorized spindles can be constantly updated, and two types of the failure mechanism, including the random failure and the fatigue failure, and four possible scenarios are taken into consideration. The integrated model can avoid unnecessary or untimely maintenance and achieve considerable economic benefit by reducing maintenance costs and raising production efficiency, which provide effective service and guidance for practitioners.

The remainder of the paper is organized as follows. Section 2 describes the CBM optimization problem and assumptions in detail, in which the failure mechanism, SPC charts, and maintenance policy are discussed. In Section 3, the integrated model for combined SPC chart design and CBM scheduling is developed. The hazard rate extracted from the PHM is used to characterize the health condition, and the SPC scheme considering four possible scenarios is established to monitor the degradation trend in real time. Then, Section 4 introduces the proposed three-stage approach for optimizing the CBM schedule upon economic considerations. In association with the PHM and the SPC charts, the average long-run cost during a renewal cycle is minimized by optimizing the upper control limit (UCL). Next, a real case of the motorized spindle is studied to verify the feasibility and practicability of the proposed approach in Section 5. Finally, conclusions are presented in the last section.

\section{Problem Statement and Assumptions}

For this research, the degradation of the motorized spindle during the long life cycle is due to both external factors, like cumulative shocks, environmental stress, etc., and internal factors, like continuous aging, etc. Therefore, the system will suffer from random failures and fatigue failures. Multiple condition features together with failure event data can be used as the health indicator to characterize the effects of degradation.

Then, the SPC chart is used as a failure diagnosis tool for monitoring the health indicator and detecting the two types of failures: the random failure, also known as the hard failure, which is self-announcing and could be detected immediately, and the fatigue failure, also known as the soft failure, which is defined to occur only when the degree of degradation reaches the preset control limits. The SPC chart scheme is shown in Figure 1, and its control limits can be usually computed following the formula [21] in Table 1 according to different SPC charts.

When combined with maintenance planning, the control limits of SPC charts can be regarded as the trigger of a maintenance action; the optimization of the CBM strategy is transformed into determining the optimal control limits in order to minimize the maintenance cost from the perspective of economic efficiency.

The integrated CBM model is developed under the assumptions as follows:

(1) The changing trend of the health indicator may not be obvious over time and increase rapidly after the system enters the wear-out stage. In order to grasp system degradation level and implement maintenance actions in time, inspections are scheduled every $\Delta$ time unit to detect the health condition periodically. The inspection time is short so that the inspection activities are considered instantaneous and nondestructive. The cost per inspection $C_{i}$ is predetermined as a constant according to the actual condition.

(2) The two types of failures correspond to two types of maintenance activities, which are the preventive replacement and the corrective replacement, respectively. To be more specific, if a hard failure occurs unexpectedly, the corrective replacement is performed instantly, and the average cost per corrective replacement $C_{c}$ covers all costs with respect to the failure and its associated problems. On the contrary, if the monitoring health indicator is detected to exceed the control limits at the inspection time, which is interpreted as a soft failure, the preventive replacement is carried out. The average 


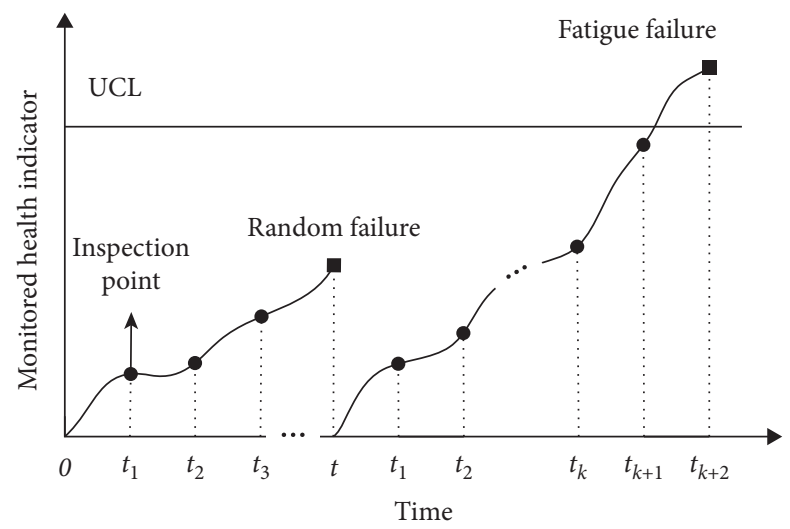

FIgUrE 1: The SPC chart scheme of motorized spindles.

TABLE 1: Characteristics of four typical SPC charts.

\begin{tabular}{|c|c|c|}
\hline Feature & Monitored statistic & Control limits \\
\hline $\begin{array}{l}\text { Shewhart } \\
\text { MA } \\
\text { CUSUM } \\
\text { EWMA }\end{array}$ & $\begin{array}{c}z_{t}=y_{t} \\
z_{t}=\left(y_{1}+y_{2}+\cdots+y_{t-w+1} / w\right) \\
z_{t}=\max \left(0, z_{t-1}+y_{t}-\mu_{0}-k\right) \\
z_{t}=y_{t}+(1-\lambda) z_{t-1}\end{array}$ & $\begin{array}{c}\mathrm{UCL}=\mu_{0}+n \sigma_{0}, \mathrm{LCL}=\mu_{0}-n \sigma_{0} \\
\mathrm{UCL}=\mu_{0}+n\left(\sigma_{0} / \sqrt{w}\right), \mathrm{LCL}=\mu_{0}-n\left(\sigma_{0} / \sqrt{w}\right) \\
\mathrm{UCL}=\mathrm{dk} \\
\mathrm{UCL}=\mu_{0}+n \sigma_{0} \sqrt{(\lambda / 2-\lambda)}, \mathrm{LCL}=\mu_{0}-n \sigma_{0} \sqrt{(\lambda / 2-\lambda)}\end{array}$ \\
\hline $\begin{array}{l}y_{t} \\
\sigma_{0} \\
W \\
D \\
\mathrm{UCL}\end{array}$ & $\begin{array}{c}\text { Monitored variable at } t \\
\text { The standard deviation of in-control data } \\
\text { Moving average width } \\
\text { Distance typically set to } 10 \\
\text { Upper control limit }\end{array}$ & $\begin{array}{cc}\mu_{0} & \text { The mean of in-control data } \\
n & \text { Control limit width } \\
k & \text { The slope of the lower arm } \\
\lambda & \text { The weighted parameter, } 0<\lambda \leq 1 \\
\text { LCL } & \text { Lower control limit }\end{array}$ \\
\hline
\end{tabular}

cost per preventive replacement $C_{p}$ covers the costs for performance maintenance, improvement, and other related operations. In general, $C_{c}>C_{p}$ since the corrective replacement is relatively more complex than the preventive replacement.

(3) After each replacement, the condition of the monitored motorized spindle will be restored to the initial state, representing that the system is under the "as good as new" maintenance strategy. In addition, it is assumed that the time for both preventive and corrective replacements is not considered in the CBM model.

\section{Condition-Based Maintenance (CBM) Model Development}

The integrated model for SPC chart design and CBM scheduling is developed from an economic point of view, whose objective is to minimize the average long-run cost considering the costs of all inspections and maintenance activities.

3.1. Proportional Hazard Model (PHM). PHM proposed by Cox [22] is a popular model which is used to describe the relationship between the degradation influence and the hazard rate. The degradation level is characterized by multiple features during lifetime operation, which are considered as covariates. The advantage of using the PHM in
CBM is that it could deduce the hazard rate considering all covariates in the current state, such as the failure event and condition monitoring factors. According to the PHM, the hazard rate at time $t$ is defined by

$$
h(t, Z(t))=h_{0}(t) \exp (\gamma Z(t)),
$$

where $h_{0}(t)$ is the baseline hazard rate at time $t$, and it will be continually modified by $Z(t)$; let $Z(t)=\left[Z_{1}(t), Z_{2}(t), \cdots\right.$ $Z_{n}(t)$ ] be a vector made up of values of $n$ condition monitoring features at time $t$, which is a time-dependent stochastic process representing the degradation evolution. And $\gamma$ is a vector made up of regression coefficients, which determines the influence weight of the monitoring features on the failure rate.

The time to failure of the electromechanical system commonly follows the Weibull distribution [23]. As for motorized spindles, their baseline hazard rate of the PHM is assumed to be derived from a two-parameter Weibull distribution, which has the expression as

$$
h_{0}(t)=\frac{\beta}{\eta}\left(\frac{t}{\eta}\right)^{\beta-1},
$$

where $\beta>0$ represents the shape parameter of the Weibull distribution and $\eta>0$ represents the scale parameter of the Weibull distribution.

Substituting the Weibull baseline hazard rate function, $Z(t)$, and $\gamma$ vectors into the PHM, Weibull proportional hazard model (WPHM) is derived as 


$$
h(t, Z(t))=\frac{\beta}{\eta}\left(\frac{t}{\eta}\right)^{\beta-1} \exp \left(\gamma_{1} Z_{1}(t)+\cdots+\gamma_{n} Z_{n}(t)\right) .
$$

Given the WPHM, then the conditional reliability can be obtained as

$$
\begin{aligned}
R(t, Z(t)) & =P(T>t \mid Z(s), 0 \leq s \leq t) \\
& =\exp \left(-\int_{0}^{t} h(s, Z(s)) \mathrm{d} s\right),
\end{aligned}
$$

where the data of failure time are denoted by $T$ and $Z(s)$ is the actual value of $Z(t)$ at time $s$.

The corresponding probability density function (PDF) is derived as

$$
f(t, Z(t))=h(t, Z(t)) R(t, Z(t))=\frac{h_{0}(t) \exp (\gamma Z(t))}{\exp \left(-\int_{0}^{t} h(s, Z(s)) \mathrm{d} s\right)} .
$$

Based on the condition monitoring system, the degradation features are acquired in real time, and the covariates could be predicted and updated, expressed as $Z(v), 0<v<\infty$. Therefore, the remaining useful life of motorized spindles can be predicted as derived in [24] by

$$
L(t)=E[T-t \mid T>t]=\int_{0}^{\infty} \exp \left(-\int_{t}^{t+\tau} h(v, Z(v)) \mathrm{d} v\right) \mathrm{d} \tau .
$$

According to the monitoring data of multiple features and the failure event data, the parameters of the WPHM can be estimated by the maximum likelihood method. Since the motorized spindle may run to failure or be maintained before failures in the actual CBM process, the failure event data collected involve both time to failure and censored time. Taking these data types into consideration, the likelihood function is given by

$$
L(\cdot)=\prod_{i=1}^{n} f\left(t_{i}, Z\left(t_{i}\right)\right) \prod_{s=1}^{m} R\left(t_{s}, Z\left(t_{s}\right)\right),
$$

where $t_{i}$ represents the value of time to failure, $t_{s}$ represents the value of censored time, $n$ is the number of failure times, and $m$ is the number of censored time. The parameter estimate of $\beta, \eta$, and $\gamma$ can be calculated by maximizing equation (7).

As a result, the hazard rate in real-time operation can be extracted by the PHM; it is adopted as the health indicator interpreting the current performance state. In the next step, SPC techniques will be applied to monitor the changing trend of the hazard rate and provide CBM decision support.

3.2. Integrated Model and Optimization. In the case of combining SPC charts with CBM scheduling for maintenance strategy optimization of motorized spindles, four possible scenarios of the integrated CBM model can occur during a renewal cycle, as shown in Figure 2. A renewal cycle is identified as the period between start of process monitoring and a maintenance activity [25], and the four different scenarios are further illustrated as follows:

Scenario $1\left(S_{1}\right)$ : the performance of the motorized spindle remains in control with no false out-of-control alarm, which is interpreted as $h(t, Z(t)) \leq \mathrm{UCL}$ When a hard failure occurs in the interval $(k \Delta,(k+1) \Delta), k=0,1, \cdots, n$, a corrective replacement is immediately implemented and restores the spindle to its initial condition.

Scenario $2\left(S_{2}\right)$ : the performance of the motorized spindle remains in control until a false out-of-control alarm is given at $(k+1) \Delta, k=0,1, \cdots, n$. A preventive replacement is performed and restores the spindle to its initial condition.

Scenario $3\left(S_{3}\right)$ : the performance of the motorized spindle shifts to the out-of-control condition in the interval $\left(k_{1} \Delta,\left(k_{1}+1\right) \Delta\right), k_{1}=0,1, \cdots, n$, which is interpreted as $h(t, Z(t)) \leq \mathrm{UCL}$. However, the SPC chart fails to capture an alert signal until the system results in a hard failure in the interval $\left(k_{2} \Delta,\left(k_{2}+1\right) \Delta\right), k_{2}=0,1, \cdots, n$. A corrective replacement is then executed and restores the spindle to its initial condition.

Scenario $4\left(S_{4}\right)$ : the performance of the motorized spindle degrades to the out-of-control condition in the interval $\left(k_{1} \Delta,\left(k_{1}+1\right) \Delta\right), k_{1}=0,1, \cdots, n-1$, and the SPC chart succeeds to detect the out-of-control signal at $k_{2} \Delta, k_{2}=k_{1}+1, \cdots, n$. A preventive replacement is carried out and restores the spindle to its initial condition.

Considering the four possible scenarios, the average long-run CBM cost $\bar{c}$ can be transformed to the expected cost over the expected length of a renewal cycle [26], which is given by

$$
\bar{c}=\frac{E(C(T))}{E(T)}
$$

where $\quad E(C(T))=\sum_{i=1}^{4} p\left(S_{i}\right) \sum_{j=1}^{m_{i}} E\left(C \mid S_{i j}\right) p\left(S_{i j}\right) \quad$ and $E(T)=\sum_{i=1}^{4} p\left(S_{i}\right) \sum_{j=1}^{m_{i}} E\left(T \mid S_{i j}\right) p\left(S_{i j}\right) \cdot p\left(S_{i}\right)$ represents the probability of the $i$ th scenario, $p\left(S_{i j}\right)$ represents the probability of the $j$ th state in the $i$ th scenario, and $m_{i}$ represents the number of states of the $i$ th scenario, which are expressed as

$$
\begin{aligned}
p\left(S_{i}\right) & =p\left(S_{i 1}\right)+p\left(S_{i 2}\right)+\cdots+p\left(S_{i m_{i}}\right), \\
m_{1} & =n+1, m_{2}=n, \\
m_{3} & =((n+1)(n+2) / 2), \\
m_{4} & =((n+1) n / 2) .
\end{aligned}
$$

The optimization objective is to obtain the minimum value of the average long-run CBM cost by setting up an optimal control limit UCL* as follows:

$$
\mathrm{UCL}^{*}=\arg \min (\bar{c}) \text {. }
$$

Taking the situation when $n=1$ for illustration, the CBM elements of each scenario are presented in Tables 2-4. 


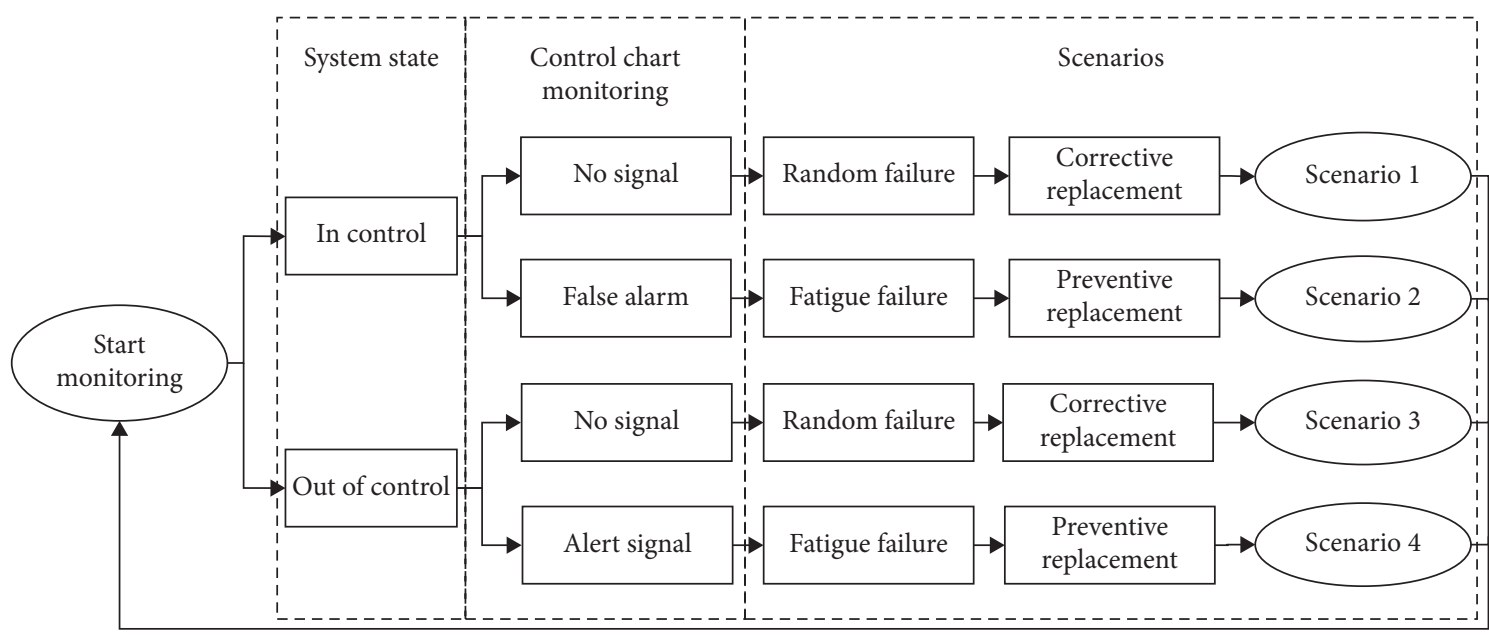

Figure 2: Four possible scenarios of the integrated CBM model.

TABLE 2: Occurrence probability of each scenario in the integrated model, $n=1$.

\begin{tabular}{|c|c|c|}
\hline Scenario & Symbol & Probability of occurrence \\
\hline \multirow[b]{2}{*}{$\left(S_{1}\right)$} & $p\left(S_{11}\right)$ & $\left(1-\exp \left(-\int_{0}^{\Delta} h_{0}(t) \int_{0}^{\infty} \varphi(z(t)) f_{Z(t)} \mathrm{d} z \mathrm{~d} t\right)\right) P(h(t) \leq \mathrm{UCL})$ \\
\hline & $p\left(S_{12}\right)$ & $\exp \left(-\int_{0}^{\Delta} h_{0}(t) \int_{0}^{D} \varphi(z(t)) f_{Z(t)} \mathrm{d} z \mathrm{~d} t\right)\left(1-\exp \left(-\int_{0}^{2 \Delta} h_{0}(t) \int_{0}^{D} \varphi(z(t)) f_{Z(t)} \mathrm{d} z \mathrm{~d} t\right)\right) P(h(t) \leq \mathrm{UCL}) P_{n}$ \\
\hline \multirow[t]{2}{*}{$\left(S_{2}\right)$} & $p\left(S_{2}\right)$ & $\exp \left(-\int_{0}^{\Delta} h_{0}(t) \int_{0}^{D} \varphi(z(t)) f_{Z(t)} \mathrm{d} z \mathrm{~d} t\right) P_{f}$ \\
\hline & $p\left(S_{31}\right)$ & $\left(1-\exp \left(-\int_{0}^{\Delta} h_{0}(t) \int_{0}^{\infty} \varphi(z(t)) f_{Z(t)} \mathrm{d} z \mathrm{~d} t\right)\right) P(h(t)>\mathrm{UCL})$ \\
\hline \multirow[t]{2}{*}{$\left(S_{3}\right)$} & $p\left(S_{32}\right)$ & $\exp \left(-\int_{0}^{\Delta} h_{0}(t) \int_{0}^{\infty} \varphi(z(t)) f_{Z(t)} \mathrm{d} z \mathrm{~d} t\right) P(h(\Delta)>\mathrm{UCL}) P_{u}\left(1-\exp \left(-\int_{0}^{2 \Delta} h_{0}(t) \int_{0}^{\infty} \varphi(z(t)) f_{Z(t)} \mathrm{d} z \mathrm{~d} t\right)\right) P(h(t)>\mathrm{UCL})$ \\
\hline & $p\left(S_{33}\right)$ & $\exp \left(-\int_{0}^{\Delta} h_{0}(t) \int_{0}^{D} \varphi(z(t)) f_{Z(t)} \mathrm{d} z \mathrm{~d} t\right)\left(1-\exp \left(-\int_{0}^{2 \Delta} h_{0}(t) \int_{0}^{\infty} \varphi(z(t)) f_{Z(t)} \mathrm{d} z \mathrm{~d} t\right)\right) P(h(t)>\mathrm{UCL}) P_{n}$ \\
\hline$\left(S_{4}\right)$ & $p\left(S_{4}\right)$ & $\exp \left(-\int_{0}^{\Delta} h_{0}(t) \int_{0}^{\infty} \varphi(z(t)) f_{Z(t)} \mathrm{d} z \mathrm{~d} t\right) P(h(\Delta)>\mathrm{UCL}) P_{d}$ \\
\hline $\bar{D}$ & & The threshold of the degradation feature, which has a relationship with \\
\hline$P_{d}$ & & The probability of the signal when the pr \\
\hline$P_{u}^{a}$ & & The probability of no signal when the process is out of control \\
\hline$P_{f}^{u}$ & & The probability of the signal when the process is in control \\
\hline$P_{n}^{J}$ & & \\
\hline
\end{tabular}

TABle 3: Expected cycle time of each scenario in the integrated model, $n=1$.

\begin{tabular}{lcr}
\hline Scenario & Symbol & Expected time per cycle \\
\hline$S_{1}$ & $E\left(T \mid S_{12}\right)$ & $\Delta-\int_{0}^{\Delta}(\Delta-t) f(t) \mathrm{d} t$ \\
$S_{2}$ & $E\left(T \mid S_{2}\right)$ & $2 \Delta-\int_{\Delta}^{2 \Delta}(2 \Delta-t) f(t) \mathrm{d} t$ \\
& $E\left(T \mid S_{31}\right)$ & $\Delta \mathrm{x}$ \\
$S_{3}$ & $E\left(T \mid S_{32}\right)$ & $\Delta-\int_{0}^{\Delta}(\Delta-t) f(t) \mathrm{d} t$ \\
& $E\left(T \mid S_{33}\right)$ & $2 \Delta-\int_{\Delta}^{2 \Delta}(2 \Delta-t) f(t) \mathrm{d} t$ \\
$S_{4}$ & $E\left(T \mid S_{4}\right)$ & $2 \Delta-\int_{\Delta}^{2 \Delta}(2 \Delta-t) f(t) \mathrm{d} t$ \\
\hline
\end{tabular}

TABLE 4: Expected cycle cost of each scenario in the integrated model, $n=1$.

\begin{tabular}{lcc}
\hline Scenario & Symbol & Expected cost per cycle \\
\hline$S_{1}$ & $E\left(C \mid S_{1}\right)$ & $\left(C_{c} p\left(S_{11}\right)+\left(C_{c}+C_{i}\right) p\left(S_{12}\right)\right) / p\left(S_{1}\right)$ \\
$S_{2}$ & $E\left(C \mid S_{2}\right)$ & $C_{p}+C_{i}$ \\
$S_{3}$ & $E\left(C \mid S_{3}\right)$ & $\left(C_{c} p\left(S_{31}\right)+\left(C_{c}+C_{i}\right) p\left(S_{32}\right)+\left(C_{c}+C_{i}\right) p\left(S_{33}\right)\right) / p\left(S_{3}\right)$ \\
$S_{4}$ & $E\left(C \mid S_{4}\right)$ & $C_{p}+C_{i}$ \\
\hline
\end{tabular}




\section{The Three-Stage Approach for CBM Optimization}

In this section, the proposed approach developed to monitor the health degradation and optimize the CBM schedule for motorized spindles is described in Figure 3. The approach is composed of three stages involving health indicator extraction, degradation condition monitoring, and CBM optimization. In order to implement this approach, the PHM is constructed, and the hazard rate is estimated, which is then applied in SPC charts as the monitoring statistic. At last, combining SPC with CBM management, an integrated optimization model is given to develop the maintenance plan and minimize the average long-run cost of maintenance by searching for the optimal control limits.

The three stages of the proposed approach are outlined as follows:

Stage 1 (construction of the PHM): the model is adopted to extract the estimation of the Stage 2 (establishment of the SPC charts): as long as the parameters of the $\mathrm{PHM}$ are estimated with the training data, the test data can be collected and plugged into the model to obtain the hazard rate as the degradation feature to be monitored. The samples of the hazard rate are then plotted on the control chart with the corresponding central line (CL) and UCL, which can be computed as Table 1 according to the selected chart type.Stage 2 (establishment of the SPC charts): as long as the parameters of the PHM are estimated with the training data, the test data can be collected and plugged into the model to obtain the hazard rate as the degradation feature to be monitored. The samples of the hazard rate are then plotted on the control chart with the corresponding central line (CL) and UCL, which can be computed as Table 1 according to the selected chart type.hazard rate by the fusion of event data and condition monitoring data. This stage is illustrated in detail as follows:

Step 1 (data acquisition). The performance of motorized spindles mainly depends on their key functional components, such as the bearings, the tool clamping system, and the rotary unions [26]. Therefore, these components would be focused by installing additional sensors, and different signals are collected comprising vibration, current, force, acoustic emission, temperature, leakage flow, etc. [19], in order to describe the degradation characteristics of the system more accurately.

Step 2 (data preprocessing and feature extraction). Using the collected life cycle signals of the monitored spindle to select the typical features in time, frequency, and time-frequency domains, approaches such as principal component analysis can be adopted for the purpose of reducing the dimension of features while keeping the information with the largest contribution to describe system degradation process.

Step 3 (health indicator estimation). Combining the extracted features from the condition monitoring system with failure event data in the lifetime of motorized spindles, the PHM is constructed, and its parameters are estimated with the training samples. Therefore, the hazard rate observation can be obtained as the health indicator for the next stage of degradation condition monitoring.

Stage 2 (establishment of the SPC charts): as long as the parameters of the PHM are estimated with the training data, the test data can be collected and plugged into the model to obtain the hazard rate as the degradation feature to be monitored. The samples of the hazard rate are then plotted on the control chart with the corresponding central line (CL) and UCL, which can be computed as Table 1 according to the selected chart type.

The chart is then adopted as a tool for anomaly detection and a trigger for maintenance activities. In particular, if a sample point plotted on the SPC chart is within the UCL, the system is considered as in-control, and the periodic inspection and daily health management are maintained. Otherwise, the system is defined as out of control, which implies that the degradation level exceeds an acceptable threshold, and a preventive replacement is performed. Meanwhile, a failure of the system could occur during both in-control and out-ofcontrol phases, which will trigger a corrective replacement.

Stage 3 (optimization of the CBM): based on the aforementioned maintenance strategy, the monitoring system integrating SPC charts and PHM is further developed for CBM optimization. Four different scenarios that can describe the possibilities in monitoring process are taken into account in order to establish the CBM model. Each scenario is ended by a maintenance activity, and the monitored system is restarted as a new cycle with an "as good as new" condition. Finally, the SPC chart is updated, and the optimal CBM schedule is obtained through minimizing the average long-run cost during a renewal cycle by optimizing the UCL.

\section{Case Study}

The proposed three-stage approach is applied to a real case of the motorized spindle in order to illustrate its strong practicability. The developing process of degradation and failure of motorized spindles are mainly blamed on bearing failures, clamping device rubbing, and rotary union loosening, which can be characterized by vibration signals [27]. Therefore, the case reported here is based on vibration signal analysis in particular.

5.1. Experimental Setup. The failure events and condition monitoring data sets are generated from the motorized spindle going through run-to-failure experiments under constant rotary speed at 4000 RPM. The experiment rig is shown in Figure 4. 


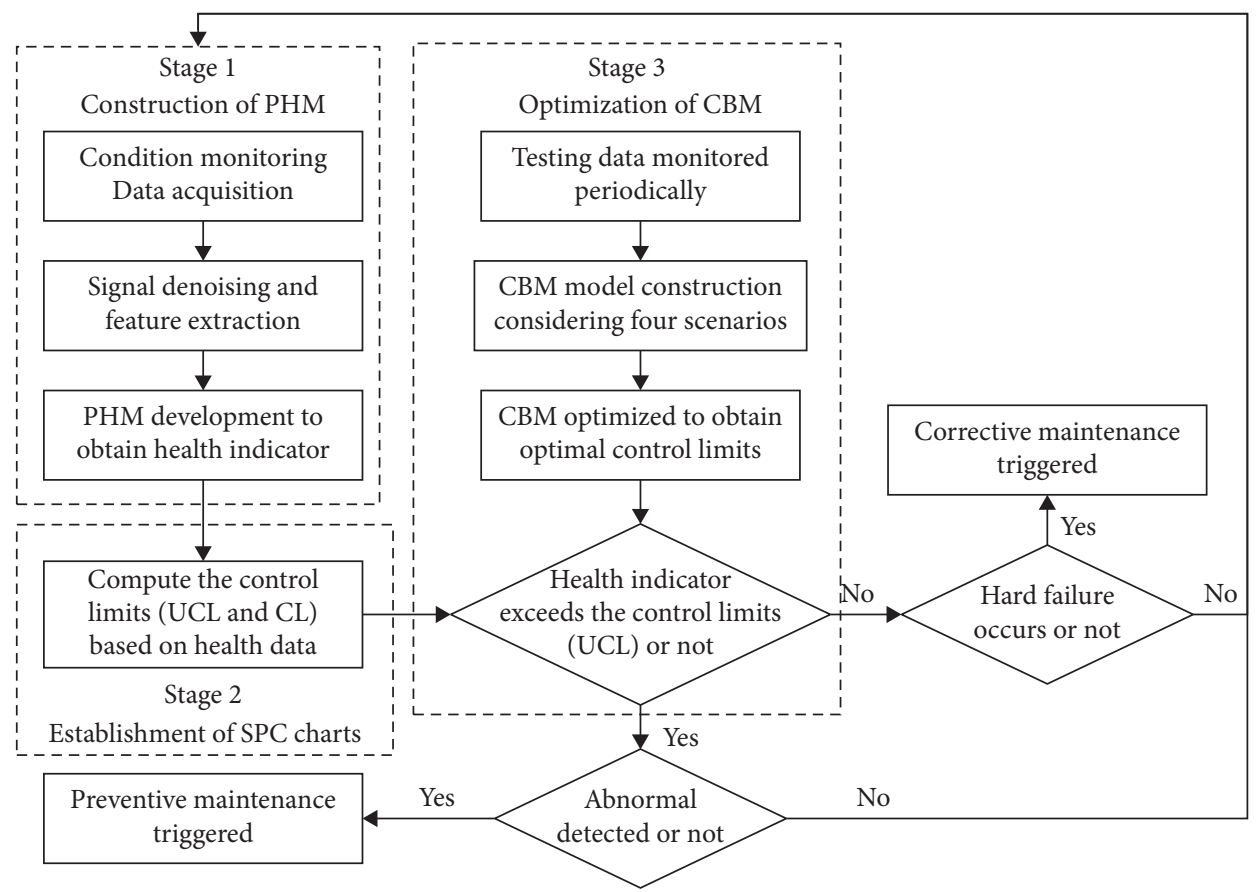

FIGURE 3: Flowchart of the proposed three-stage approach.

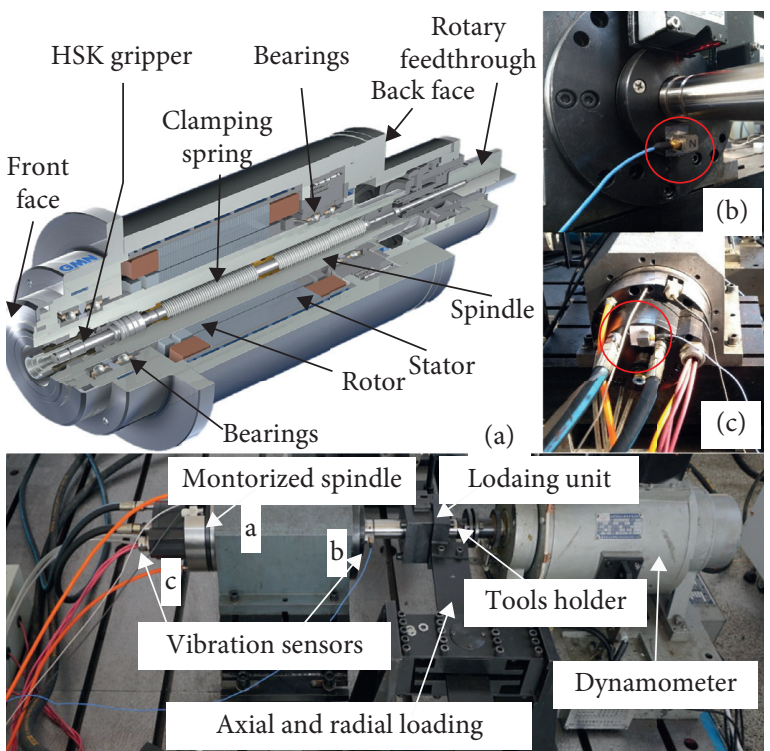

FIGURE 4: The experiment rig for motorized spindle monitoring.

The experimental setup is designed considering the structure of the motorized spindle, which is shown in Figure 4(a); the spindle can be subjected to the cutting torque provided by the dynamometer joined to the tool holder with the couplings and the biaxial cutting forces provided by the axial and radial loading systems. The spindle is kept running 12 hours per day except for irregular equipment adjustment and unexpected failures.

Based on experimental requirements, two PCB 356A15 triaxial high sensitivity, ceramic shear ICP accelerometers are installed on the front face and the back face of the motorized spindle, respectively. The specific placement is circled in Figures 4(b) and 4(c), and the vibration signal is collected every 4 hours by ADLINK's USB-24054-CH, 24bit, $128 \mathrm{kS} / \mathrm{s}$ dynamic signal acquisition. The test and the condition monitoring system are controlled by LabVIEW program.

5.2. Feature Extraction and PHM Construction. The training data collected include time of inspections, time to failure, censored time, and vibration signal. Each inspection lasts at least 30 seconds, which contains 150,000 vibration signals with the sampling rate of $5 \mathrm{kHz}$. A total of 246 inspections spanning over 1000-hour operation test on the tested motorized spindle are conducted. During the whole experimental period, 7 hard failures and 5 suspensions are observed. Among them, the 7 failures are considered as unexpected functional failures, and the 5 suspensions are due to adjustments of experimental equipment.

Only the $x$-axial vibration measurements were used for analysis, and three commonly used time-domain features are extracted from the lifetime vibration data of the tested spindle. The technicians believe that the selected features can contain essential information to interpret the specific failure well [28]. The features as derived in [29] are summarized in Table 5.

PHMs with different features are fitted, and K-S goodness-of-fit tests are performed to obtain a better model. The parameters and the test statistics of hazard rate functions are estimated and listed in Table 6. The traditional Weibull distribution without incorporating any feature is also fitted, and its result is shown in Table 6 as Model 1 .

It implies in Table 6 that the significance of RMS in the PHM is greater than kurtosis and crest factor. In addition, the fitting effect of the PHM integrating with condition 
TABLE 5: Summary of selected time-domain features.

\begin{tabular}{lcc}
\hline Feature & Definition & Formula \\
\hline RMS & Root mean square, the square root of the mean square & $\sqrt{(1 / N) \sum_{i=1}^{N}\left(x_{i}-\bar{x}\right)^{2}}$ \\
$\begin{array}{l}\text { Kurtosis } \\
\text { Crest factor }\end{array}$ & A measure of the vibratory magnitudes of the probability distribution in the time domain & $(1 / N) \sum_{i=1}^{N}\left(x_{i}-\bar{x} / \mathrm{RMS}\right)^{4}$ \\
\hline
\end{tabular}

TABLE 6: Estimation results for the selected PHMs.

\begin{tabular}{|c|c|c|c|c|c|c|c|}
\hline \multirow{2}{*}{ Model } & \multicolumn{2}{|c|}{$h_{0}(t)$} & \multicolumn{3}{|c|}{$\gamma_{i}$} & \multirow{2}{*}{ K-S } & \multirow{2}{*}{$p$} \\
\hline & $\beta$ & $\eta$ & $\gamma_{1}(\mathrm{RMS})$ & $\gamma_{2}$ (kurtosis) & $\gamma_{3}$ (crest factor) & & \\
\hline 1 & 1.7634 & 162.2420 & - & - & - & 0.3028 & 0.4551 \\
\hline 2 & 2.0046 & 151.3602 & 1.0768 & - & - & 0.2190 & 0.8255 \\
\hline 3 & 2.2251 & 124.7858 & - & 0.0822 & - & 0.2595 & 0.6442 \\
\hline 4 & 2.0661 & 168.8726 & - & - & 0.1218 & 0.2846 & 0.5302 \\
\hline
\end{tabular}

monitoring features is better than the traditional Weibull distribution. Since the PHM with RMS has the lowest K-S statistic of 0.2190 and the highest $p$ value of 0.8255 comparing with the other models in the table, the PHM with RMS is selected for further degradation monitoring and maintenance decision of the motorized spindle, and its parameters are estimated as $\beta=2.0046 \eta=151.3602$, and $\gamma_{1}=1.0768$.

5.3. SPC Establishment and Degradation Monitoring. For a monitored renewal cycle of the motorized spindle, a life cycle of 372 hours with 93 inspections in total is adopted to obtain the 93 testing values of RMS as the lifetime features, which are shown in Figure 5(a). Then, the set of RMS is plugged into the PHM obtained from the training data set in Section 5.2 , and the hazard rate is calculated and plotted in Figure 5(b).

The hazard rate set of the testing cycle is then used as monitored health indicators. The SPC charts introduced in Section 2 are constructed as supports for anomaly detection and CBM optimization. The Shewhart I-chart and the EWMA chart are taken as examples, which are shown in Figures 6(a) and 6(b). In the case of monitoring the hazard rate, only UCL and CL are essential. The red solid lines represent the UCLs of the charts, the green-dotted lines represent the CLs of the charts, the black dots are corresponding statistics derived from the testing data of the hazard rate, and the marked purple circles are degradation states determined to be out of control.

To be more specific, the two charts are established following the rules in Table 1. As for the Shewhart I-chart in Figure 6(a), the estimated process mean $\mu_{0}=0.0219$, the estimated process standard deviation $\sigma_{0}=0.0013$, $\mathrm{UCL}=0.0258, \mathrm{CL}=0.0219$, and the control limit width $n=3$, and the process is determined to be out of control at the 62 nd inspection. Figure 6(b) depicts the results of the EWMA chart, whose weight parameter is set to be $\lambda=0.25$, the UCL is stable at $\mathrm{UCL}=0.0234$, and the process is determined to be out of control at the 60th inspection.
Since the Shewhart I-chart monitors the performance of the motorized spindle only based on observed data at individual inspections, it is less sensitive in detecting degradation with small and moderate change trends in comparison with the EWMA chart [30]. However, in the case of monitoring the hazard rate of the tested spindle, the results of the EWMA chart show little significant deviation comparing to the Shewhart I-chart, which is mostly the consequence of using the hazard rate from the proposed PHM as the monitoring statistic. The model could compromise multiple information based on time series in order to eliminate autocorrelation in the process and reduce the influence of the random error. As a result, the two types of charts' abilities for gradual degradation detection are satisfactory.

5.4. CBM Schedule Optimization. To build the final CBM model, the established SPC chart is keen to be updated and optimized by finding optimal UCL* , which is used to trigger corresponding maintenance actions so that the inspection and maintenance cost can be minimized.

In the current case, the tested spindle is inspected every $\triangle=4$ hours, the cost of each inspection $C_{i}$ is $¥ 30$. The preventive replacement cost $C_{p}$ is $¥ 500$, and the corrective replacement cost $C_{c}$ is $¥ 6000$. To simplify the analysis, $P_{d}$ and $P_{f}$ are set to be 0 , and $P_{u}$ and $P_{n}$ are set to be 1 , which reduce the four scenarios to two for the control chart. Finally, the CBM model can be interpreted as follows:

Scenario $1\left(S_{1}\right)$ : the motorized spindle remains in control until a hard failure occurs in the interval $(4 k, 4(k+1))$. A corrective replacement is implemented and restores the spindle to its initial condition.

Scenario $2\left(S_{2}\right)$ : the motorized spindle degrades to the out-of-control condition in the interval $(4 k, 4(k+1))$, and the SPC chart succeeds to detect it at $4(k+1)$. A preventive replacement is carried out and restores the spindle to its initial condition.

As a result, the long-run cost rate is obtained through the following formula: 


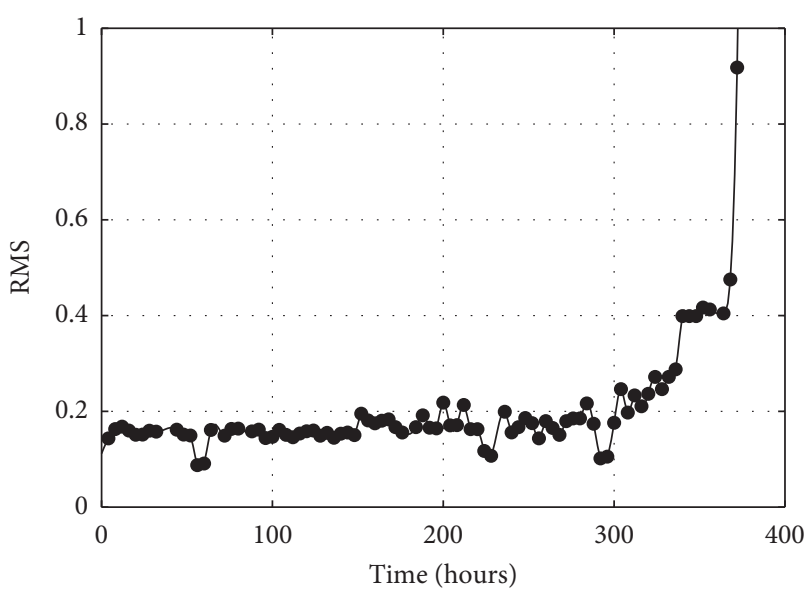

(a)

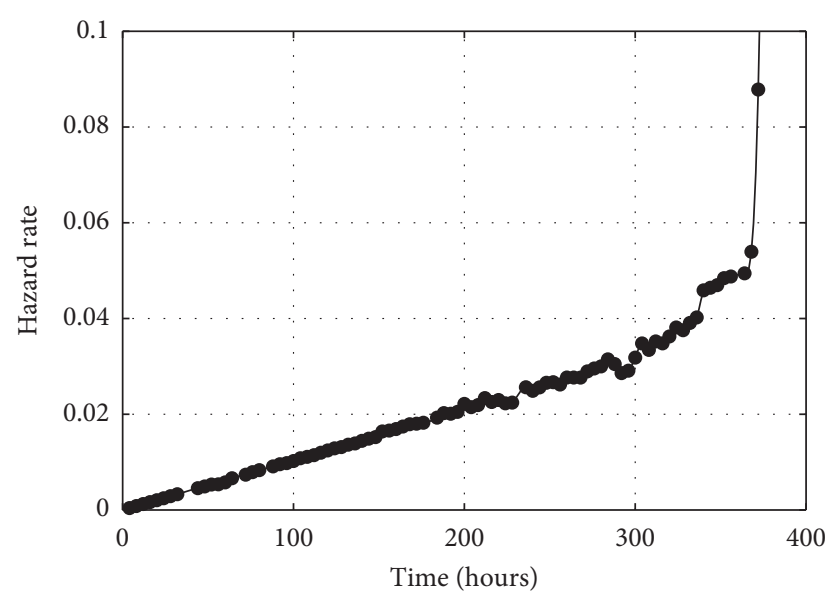

(b)

FIGURE 5: Lifetime features for the tested motorized spindle. (a) RMS. (b) The hazard rate estimates.

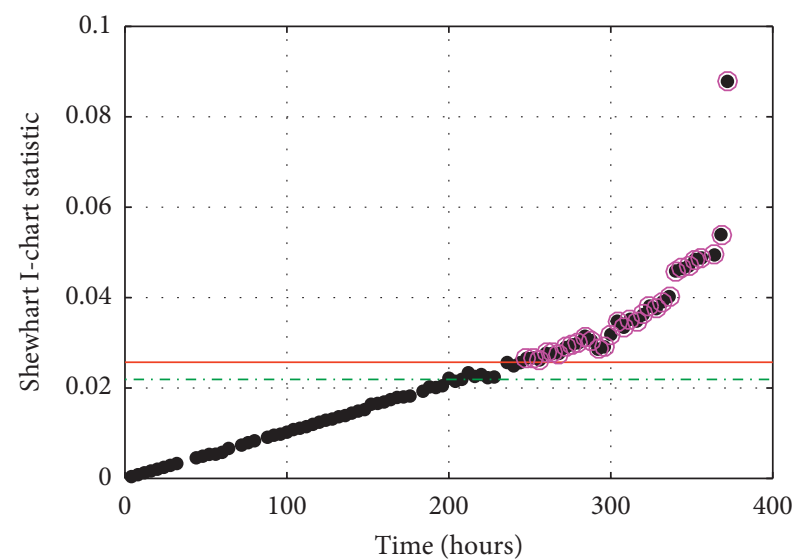

- In-control sample - UCL

(-) Out-of-control sample -.. CL

(a)

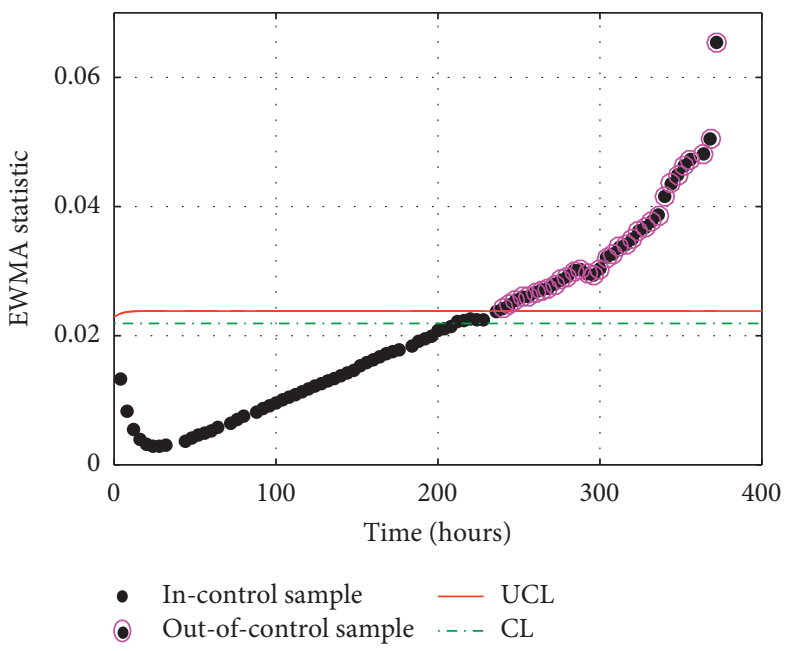

(b)

Figure 6: Two types of SPC charts for the tested motorized spindle. (a) The Shewhart I-chart. (b) The EWMA chart.

$$
\bar{c}=\frac{\sum_{0}^{93}\left(C_{c}+k C_{i}\right) p_{k}\left(S_{1}\right)+\sum_{0}^{93}\left(C_{p}+(k+1) C_{i}\right) p_{k}\left(S_{2}\right)}{\sum_{0}^{93}\left(4(k+1)-\int_{4 k}^{4(k+1)}(4(k+1)-t) f(t) \mathrm{d} t\right) p_{k}\left(S_{1}\right)+\sum_{0}^{93} 4(k+1) p_{k}\left(S_{2}\right)} .
$$

Based on the vibration signals at each inspection of the degradation process, RMS is found to follow the normal distribution with parameters $\mu(t)$ and $\sigma(t)$. The changes of the two parameters with time are studied with respect to the 93 inspections, which are expressed as

$$
\begin{aligned}
& \mu(t)=0.11 \exp \left(1.14 \times 10^{-3} t\right)+8.96 \times 10^{-16} \exp (0.09 t) . \\
& \sigma(t)=0.04 \exp \left(2.65 \times 10^{-4} t\right)+1.87 \times 10^{-8} \exp (0.04 t) .
\end{aligned}
$$

Therefore, the probability that the hazard rate exceeds UCL at time $t$ is derived as

$$
P(h(t)>\mathrm{UCL})=1-\Phi\left(\frac{D-\mu(t)}{\sigma(t)}\right) .
$$

Through optimization of equation (11), the optimal CBM schedule is finally obtained with $\mathrm{UCL}^{*}=0.0524$, and the minimal long-run cost rate is $\bar{c}=15.1942$, which is shown as the red-marked circle in Figure 7(a). According to equation (3), the RMS threshold versus the testing time of the motorized spindle can be obtained and depicted in Figure 7(b). The result also has certain directive significance in practical application because the preventive replacement can be simply triggered when the RMS observation at the inspection exceeds the RMS threshold. 


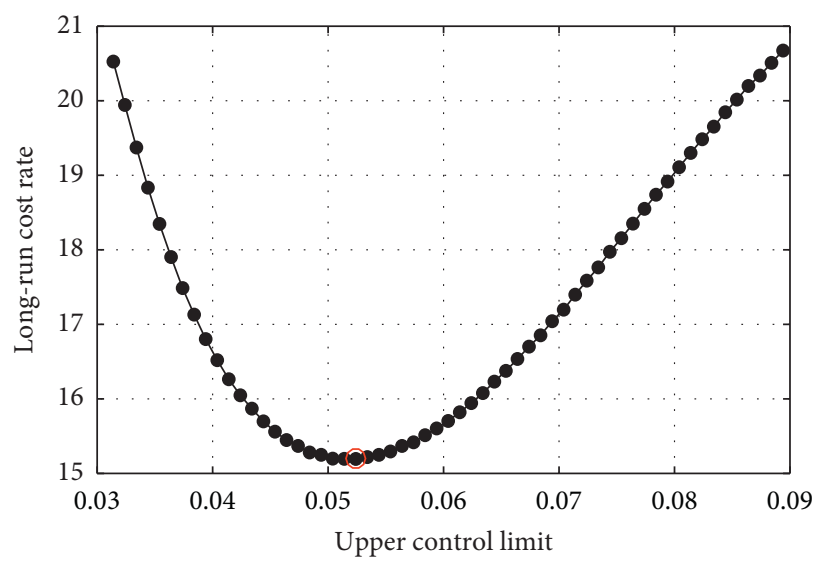

(a)

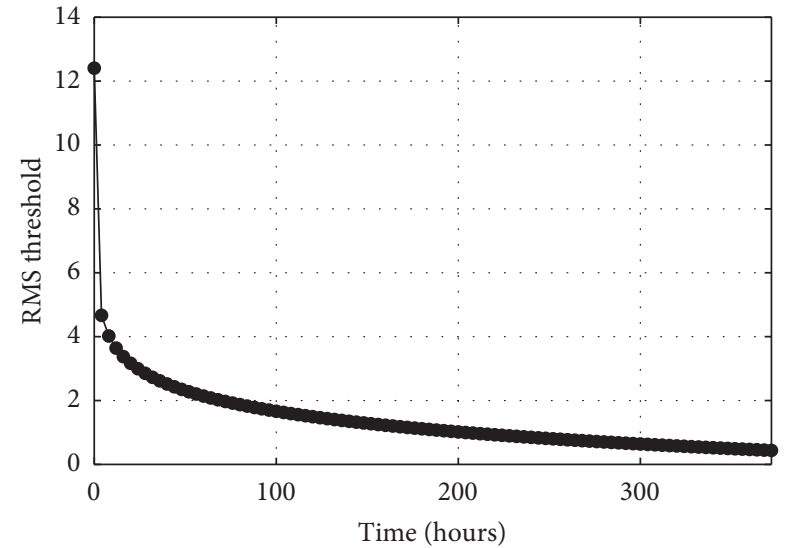

(b)

FIgURE 7: The CBM optimization results. (a) The average long-run cost rate versus UCL. (b) The RMS threshold versus time.

\section{Conclusions}

The proposed three-stage approach in this paper is committed to fusing various degradation features, monitoring the degradation process, and developing the optimal CBM plans for motorized spindles. The combined effect of condition features degrading over time together with failure events is interpreted by the PHM, and SPC charts are then introduced to monitor the changing trend of the hazard rate obtained from the PHM. Application of the SPC charts with periodic hazard rate inspections helps in CBM modelling, and four possible scenarios are defined to constitute the maintenance model. At last, the CBM plan with minimum long-run cost is obtained by seeking for the optimal UCL.

The feasibility and practicability of the approach are proved by a case study using the actual spindle vibration data. The analysis results indicate that the PHM contributes to describing the degradation states of the motorized spindle more accurately, and three different time-domain features are extracted from the raw vibration signals and are used as covariates in the PHM; RMS is verified to have the best fitting effect of the experimental data.

The proposed approach allows not only maintenance optimization but also accurate characterization of health degradation. It can help maintenance technicians and managers reduce breakdown time, delete unnecessary maintenance actions, and minimize maintenance costs in an intuitive and efficient way. The approach in this paper can also be applied for the motorized spindle operating in CNC machine tools for intelligent manufacturing in order to achieve better performance and a longer life of the whole manufacturing supply chain.

\section{Data Availability}

No data were used to support this study.

\section{Conflicts of Interest}

The authors declare that there are no conflicts of interest regarding the publication of this paper.

\section{Acknowledgments}

This research was supported by the Fundamental Research Funds for the Central Universities (Grant no. 3072020CFJ0202).

\section{References}

[1] T. Liu, W. Gao, D. Zhang et al., "Analytical modeling for thermal errors of motorized spindle unit," International Journal of Machine Tools and Manufacture, vol. 112, pp. 5370, 2017.

[2] S. Alaswad and Y. Xiang, "A review on condition-based maintenance optimization models for stochastically deteriorating system," Reliability Engineering \& System Safety, vol. 157, pp. 54-63, 2017.

[3] Y. Lei, N. Li, L. Guo, N. Li, T. Yan, and J. Lin, "Machinery health prognostics: a systematic review from data acquisition to RUL prediction," Mechanical Systems and Signal Processing, vol. 104, pp. 799-834, 2018.

[4] D. Shrivastava, M. S. Kulkarni, and P. Vrat, "Integrated design of preventive maintenance and quality control policy parameters with CUSUM chart," The International Journal of Advanced Manufacturing Technology, vol. 82, no. 9-12, pp. 2101-2112, 2016.

[5] Y. Li, E. Pan, and Z. Chen, "Considering machine health condition in jointly optimizing predictive maintenance policy and X-bar control chart," in Proceedings of the International Conference On Grey Systems And Intelligent Services (GSIS 2017, pp. 328-337, Stockholm, Sweden, August 2017.

[6] A. Farahani, H. Tohidi, and A. Shoja, "An integrated optimization of quality control chart parameters and preventive maintenance using Markov chain," Advances in Production Engineering \& Management, vol. 14, no. 1, pp. 5-14, 2019.

[7] S. P. G. F. d. S. Lampreia, J. F. G. Requeijo, J. A. M. Dias, V. M. Vairinhos, and P. I. S. Barbosa, "Condition monitoring based on modified CUSUM and EWMA control charts," Journal of Quality in Maintenance Engineering, vol. 24, no. 1, pp. 119-132, 2018.

[8] P. Charongrattanasakul and A. Pongpullponsak, "Minimizing the cost of integrated systems approach to process control and maintenance model by EWMA control chart using genetic algorithm," Expert Systems with Applications, vol. 38, no. 5, pp. 5178-5186, 2011. 
[9] M. A. Ardakan, A. Z. Hamadani, M. Sima et al., "A hybrid model for economic design of MEWMA control chart under maintenance policies," The International Journal of Advanced Manufacturing Technology, vol. 83, no. 9-12, pp. 2101-2110, 2016.

[10] J. Zhong and Y. Ma, "An integrated model based on statistical process control and maintenance for two-stage dependent processes," Communications in Statistics Simulation \& Computation, vol. 46, no. 1, pp. 106-126, 2017.

[11] H. Rasay, M. S. Fallahnezhad, and Y. Zare Mehrjerdi, “An integrated model for economic design of chi-square control chart and maintenance planning," Communications in Statistics - Theory and Methods, vol. 47, no. 12, pp. 2892-2907, 2018.

[12] W. H. Zhou and G. L. Zhu, "Economic design of integrated model of control chart and maintenance management," Mathematical \& Computer Modelling, vol. 47, no. 11-12, pp. 1389-1395, 2008.

[13] Z. Mehrafrooz and R. Noorossana, "An integrated model based on statistical process control and maintenance," Computers \& Industrial Engineering, vol. 61, no. 4, pp. 1245-1255, 2011.

[14] H. Yin, G. Zhang, H. Zhu et al., "An integrated model of statistical process control and maintenance based on the delayed monitoring," Reliability Engineering System Safety, vol. 133, pp. 323-333, 2015.

[15] C. W. Zhang, M. Xie, J. Y. Liu, and T. N. Goh, "A control chart for the Gamma distribution as a model of time between events," International Journal of Production Research, vol. 45, no. 23, pp. 5649-5666, 2007.

[16] Y. He, F. Liu, J. Cui et al., "Reliability-oriented design of integrated model of preventive maintenance and quality control policy with time-between-events control chart," Computers \& Industrial Engineering, vol. 129, pp. 228-238, 2019.

[17] R. Li and X. Zhang, "Preventive maintenance interval optimization for continuous multistate systems," Mathematical Problems in Engineering, vol. 2020, Article ID 2942940, 10 pages, 2020.

[18] Y. Li, Z. Chen, and E. Pan, "Joint economic design of CUSUM control chart and age-based imperfect preventive maintenance policy," Mathematical Problems in Engineering, vol. 2018, pp. 9246372-11, 2018.

[19] H. Cao, X. Zhang, and X. Chen, "The concept and progress of intelligent spindles: a review," International Journal of Machine Tools and Manufacture, vol. 112, pp. 21-52, 2017.

[20] F. Kadri, F. Harrou, S. Chaabane, Y. Sun, and C. Tahon, "Seasonal ARMA-based SPC charts for anomaly detection: application to emergency department systems," Neurocomputing, vol. 173, pp. 2102-2114, 2016.

[21] D. C. Montgomery, Introduction to Statistical Quality Control, John Wiley \& Sons, New York, NY, USA, 2012.

[22] D. R. Cox, "Regression models and life-tables," in Breakthroughs in Statistics, S. Kotz and N. L. Johnson, Eds., pp. 527-541, Springer, Berlin, Germany, 1992.

[23] J. I. McCool, Using the Weibull Distribution: Reliability, Modelling, and Inference, John Wiley \& Sons, New York, NY, USA, 2012.

[24] H. Liao, W. Zhao, and H. Guo, "Predicting remaining useful life of an individual unit using proportional hazards model and logistic regression model," in Proceedings Of the Annual Reliability And Maintainability Symposium (RAMS '06), pp. 127-132, Newport Beach, CA, USA, January 2006.
[25] B. Liu, Z. Liang, A. K. Parlikad, M. Xie, and W. Kuo, "Condition-based maintenance for systems with aging and cumulative damage based on proportional hazards model," Reliability Engineering \& System Safety, vol. 168, pp. 200-209, 2017.

[26] R. Neugebauer, J. Fischer, and M. Praedicow, "Conditionbased preventive maintenance of main spindles," Production Engineering, vol. 5, no. 1, pp. 95-102, 2011.

[27] D. Goyal and B. S. Pabla, "Condition based maintenance of machine tools-A review," CIRP Journal of Manufacturing Science and Technology, vol. 10, pp. 24-35, 2015.

[28] C. De Castelbajac, M. Ritou, S. Laporte, and B. Furet, "Monitoring of distributed defects on HSM spindle bearings," Applied Acoustics, vol. 77, pp. 159-168, 2014.

[29] S. Güneş, M. Dursun, K. Polat et al., "Sleep spindles recognition system based on time and frequency domain features," Expert Systems with Applications, vol. 38, no. 3, pp. 24552461, 2011.

[30] W. A. Jensen, L. A. Jones-Farmer, C. W. Champ, and W. H. Woodall, "Effects of parameter estimation on control chart properties: a literature review," Journal of Quality Technology, vol. 38, no. 4, pp. 349-364, 2006. 